\title{
A Novel Design of Efficient Tracking System and String Level Monitoring System for PV Power Plant
}

\author{
Wonchul Choi ${ }^{1}$, Hyukjae Woo $^{2}$ and Choongchae $\mathrm{Woo}^{3}$ \\ ${ }^{1}$ Department of Electronic Engineering, Hanseo Univ, Seosan, Korea \\ ${ }^{2} S D N$ Company Ltd, Gwangju, Korea \\ ${ }^{3}$ Department of Electronic Engineering, \\ Hanseo Univ, Seosan, Korea \\ ${ }^{1}$ comling2497@gmail.com, ${ }^{3}$ Woo9@hanseo.ac.kr
}

\begin{abstract}
These days photovoltaic (PV) power generation is the most important part of renewable energy. Solar tracker are also rising in popularity. Solar tracking system increases the amount of energy produced from a fixed amount of installed power generating capacity, but system cost is also increased. This cost is bottleneck of increasing demand of solar tracker.

In this thesis, A novel design of cost efficient tracking system using photovoltaic cell and centralized control is proposed. A single PV sensor unit is used for detecting azimuth of solar and control all individual tracker with wireless sensor network in entire PV generating system. So we called a new system as centralized tracking system using a single PV cell (CTSUP). CTSUP provides a competitive performance compared with a conventional dual axis tracking system and reduce the cost of tracking system.

Moreover, string level power monitoring system(SLPMS) is also proposed to decrease labor cost of operation and management of PV power generating system. SLPMS not only provide a measurement result of power amount of a single string but also diagnostic of $P V$ panel problems in PV power generating systems. Combinations of proposed CTSUP and SLPMS can reduce both CAPEX and OPEX of PV power generation system.
\end{abstract}

Keywords: Tracking System, Power monitoring System, PV Power Generation Plant

\section{Introduction}

The solar energy had been used a renewable energy and set to become increasingly important in the longer term, for providing the electricity. The most abundant energy in the universe is obtained from sun. Hence it is the challenge for the present generation to generate maximum electricity by using solar power although many developments were made to collect the solar energy, but unable to utilize the maximum power released from sun.

To utilize the maximum power released from sun, servo control tracking system can be used[1-2]. Solar servo control tracking system which consists of remotely controlling and monitoring a solar module assembly for smooth driven according to the solar azimuth. However conventional tracking system has a some problems described as below:

(a) The existing solar tracking device utilizes DC motor and a worm gear to give a driving force to the photo conductive cell (cds) and a solar module assembly by measuring luminance to detect rotation angle of the tracking device.

Received (January 3, 2018), Review Result (March 5, 2018), Accepted (March 12, 2018) 
(b) The light sensors like cds are arranged at the top of the solar module assembly for detecting the solar azimuth which increases cost, and it is very difficult to detect when weather is changed. Even measured azimuth will have large error range due to the cds.

(c) DC motor is not sufficient to drive more than six module assemblies as it has less driving torque, hence conversion of AC $220 \mathrm{~V}$ to $\mathrm{DC}$ power is required that increases the cost.

(d) It is difficult to control the driven angle of solar module assembly as the angle is measured by counting the number of pulses generated per one revolution using the proximity sensor.

(e) To maintain the fixed horizontal position of solar module assembly the artificial manipulation is required to prevent damage from natural calamities like typhoon, heavy snow fall etc.

(f) To inspect the solar module assembly always an operator must visit the site it increases the maintenance cost, as the system is not equipped with monitoring[3-4].

Solar tracking system can increase the amount of energy produced from a fixed amount of installed power generating capacity, but above drawbacks of conventional solar tracking system prevent increasing demand of solar tracker.

One of most important technique for management of PV power plant is power measurement and monitoring system. In these days, there are many researches for efficient management of PV power generation systems[3-4]. In the conventional power monitoring system, the power from every single module is measured and transmit the information via wired or wireless communication channel to monitoring center. It increasing the cost of monitoring system. On the other hand, if overall power generated in PV power generation plant is just measured and monitored, cost can be reduced dramatically but there are no way to find a failure of a PV panel.

Solar tracking system can increase efficiency of power generation in PV cell and Power monitoring system can increase efficiency of management of PV power plant. However, both conventional solar ftracking system and power monitoing system have a drawbacks. In this dissertation, the drawbacks are eliminated by new design.

\subsection{Central Method of Pyranometer Measurement Device}

The present invention is the remedy for the above disadvantages, which provides a remotely servo controlling and monitoring the solar module assembly according to the solar azimuth measured by single solar cell sensor and solar module assembly that consists of solar panels arranged in 4X4 matrix form to achieve high electric power generation with less cost. The solar servo control tracking device also comprises of:

(a) Integrated control device that consist single solar cell sensor unit detects the luminance of sunrays according to the solar azimuth.

(b) Control panel that transmits control signals remotely with respect to maximum solar azimuth calculated by comparing with sunrays incident on the solar cell sensor unit and solar azimuth measured in real time. Solar tracking device that includes a solar tracking device controller remotely that receives the control signals through wireless network.

(d) High torque driving unit driven by $\mathrm{AC}$ single phase inductor to generate high torque with respect to the control signal.

(e) The operating angle of sensor unit is installed to high torque driving unit, to track the sun according to the control signal[5-6].

The single tracking controller with illumination Sensor can measure accurately the light strength for solar azimuth angle and can define the driving angle command using the optimal algorithm of solar azimuth angle. Also, the angle command is transmitted to the 
tracker controllers using the wireless communication unit and irradiation sensor, wind sensor, remote unit, track controller, elevation driver. The proposed solar tracking systems are totally automation for this purpose design and programing the drivers, sensors, measurement and monitoring system for reducing cost and give the best output [7].

In the proposed tracking controller, a single PV sensor unit is used for detecting azimuth of solar and control all individual tracker with wireless sensor network in entire PV generating system. So we called a new system as centralized tracking system using a single PV cell (CTSUP) [8].

Moreover, string level power monitoring system(SLPMS) is also proposed to decrease labor cost of operation and management of PV power generating system. SLPMS not only provide a measurement result of power amount of a single string but also diagnostic of PV panel problems in PV power generating systems [9].

In the conventional power monitoring system, the power from every single module is measured and transmit the information to monitoring center. It increasing the cost of monitoring system. On the other hand, if overall power generated in PV power generation plant is just measured and monitored, cost can be reduced dramatically but there are no way to find a failure of a PV panel.

There SLPMS measure a generated power from a single string, so the cost of power monitoring system can be reduced. SLPMS measured a generated from strings and compared the power generated from individual string. Therefore, if there are a failure of a PV cell in the string, the power generated from the string is little bit lower than other that of string. So, SLPMS can detect a failure of PV cell. this failure diagnosis function of SLPMS can be reduce OPEX of PV power generation plant. Combinations of proposed CTSUP and SLPMS can reduce both CAPEX and OPEX of PV power generating system.

A full detail of this dissertation is given as follows. This introductory chapter presents the motivation, contributions and outline of the dissertation. In Chapter 2, the general component of PV power generation plant and technical issue in PV power generation plant is present. In Chapter 3, A novel solar tracking system using PV cell as a solar sensor is proposed and described. The string level measurement and monitoring system is proposed in Chapter 4. Finally, Chapter 5 presents the conclusions and research topics for future.

\section{Design of Solar Sensor}

A solar servo control tracking device using a pyranometer is disclosed. The device includes: a solar panel; a tracker having a solar panel driving motor driving the solar panel; a tracker controller controlling the tracker; first pyranometers measuring a solar azimuth in east-west (EW) and detecting solar radiation of $380 \mathrm{~nm}$ to $840 \mathrm{~nm}$ wavelengths; second pyranometers measuring a solar azimuth in NS and detecting solar radiation; and a third pyranometer measuring a solar azimuth in the CE and detecting solar radiation. The tracker controller includes: signal amplifiers amplifying detection signals in three azimuths by the pyranometers; signal processors filtering the amplified detection signals by limiting a voltage; and a main control microcomputer outputting a control signal to the solar panel driving motor to stop the solar panel when the solar radiations are same and the solar radiations in one direction are maximum.

The increasing demand for energy, the continuous reduction in existing sources off oil fuels and the growing concern regarding environment pollution, have pushed mankind to explore new technologies for the production of electrical energy using clean, renewable sources, such as solar energy, wind energy, etc. Among the non-conventional, renewable energy sources, solar energy affords great potential for conversion into electric power, able to ensure an important part of the electrical energy needs of the planet.

The conversion of solar light into electrical energy represents one of the most promising and challenging energetic technologies, in continuous development, being 
clean, silent and reliable, with very low maintenance costs and minimal ecological impact. Solar energy is free, practically inexhaustible, and involves no polluting residues or greenhouse gases emissions. The conversion principle of solar light into electricity, called Photo-Voltaic or PV conversion, is not very new, but the efficiency improvement of the PV conversion T. Tudorachetal. Design of a Solar Tracker System for PV Power Plants equipment is still one of top priorities for many academic and/or industrial research groups all over the world. Among the proposed solutions for improving the efficiency of PV conversion, we an mention solar tracking the optimization of solar cell configuration and geometry new materials and technologies etc.

The global market for PV conversion equipment has shown an exponential increase over the last years, showing a good tendency for the years to come. Physically, a PV panel consists of a flat surface on which numerous p-n junctions are placed, connected together through electrically conducting strips. The PV panel ensures the conversion of light radiation into electricity and it is characterized by a strong dependence of the output power on the incident light radiation.

As technology has evolved, the conversion efficiency of the PV panels has increased steadily, but still it does not exceed $13 \%$ for the common ones. The PV panels exhibits a strongly non-linear I-V (current - voltage) characteristic and a power output that is also non-linearly dependant on the surface insolation. In the case of solar light conversion into electricity, due to the continuous change in the relative positions of the sun and the earth, the incident radiation on a fixed PV panel is continuously changing, reaching a maximum point when the direction of solar radiation is perpendicular to the panel surface. In this context, for maximal energy efficiency of a PV panel, it is necessary to have it equipped with a solar tracking system.

\subsection{Central Method of Pyranometer Measurement Device}

The present invention relates to a solar servo control tracking device for a solar photovoltaic generation, and more particularly, the solar to a solar servo control tracking device using a pyranometer. In general, solar light contains ultraviolet light of 3\%, visible light of $43 \%$, and infrared light of $54 \%$. The wavelength with the highest energy density for solar photovoltaic generation is $380 \mathrm{~nm}$ to $780 \mathrm{~nm}$ of visible light and the wave of the next highest energy density is $840 \mathrm{~nm}$ of near infrared light wavelength of which is relatively short among infrared light.

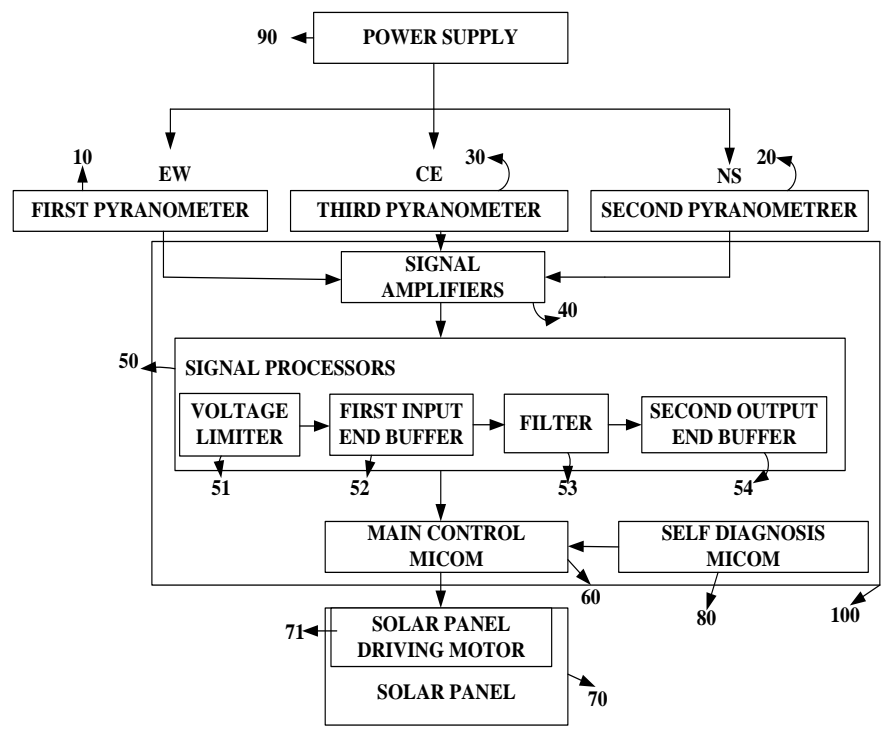

Figure 1. Block Diagram Illustrating a Solar Servo Control Tracking Device Using Pyranometer 
A solar cell (photovoltaic cell) performs energy conversion from sunlight to electric energy mainly using the wavelength of the visible light $380 \mathrm{~nm}$ to $780 \mathrm{~nm}$ and the wavelength of the near infrared light $840 \mathrm{~nm}$. An existing device for detecting a sunlight incident angle is settled to measure the incident angle where solar radiation is maximized using a cadmium sulfide (cds) light sensor. However, the detection of an incident angle of sunlight using the cds light sensor has the following disadvantages. First, since only some of $380 \mathrm{~nm}$ to $450 \mathrm{~nm}$ (low band visible light) among wavelengths of $380 \mathrm{~nm}$ to $780 \mathrm{~nm}$ of visible light having high solar energy density is detectable and detection sensitivity becomes inferior due to non-linearity with respect to an incident angle of the sunlight, it is difficult to precisely detect sunlight. Since detection output is weak in cloudy or very changeable weather due to the detection output exhibiting a second order function characteristics, it is difficult to detect the precise incident angle of sunlight so that it is hard to improve the efficiency of solar photovoltaic generation. Second, since the detected incident angle of sunlight is restricted due to a triangular pyramid shape of the cds light sensor, azimuth angles of sunlight with respect to east, west, south, and north cannot be detected. Due to this, two cds light sensors are used. However, even in the case when the two cds light sensors are used, it is difficult to track sunlight in the very changeable weather.

The present invention has been made in view of the above problems, and it is an object of the present invention to provide a solar servo control tracking device using pyranometer for precisely detecting energy density of wavelengths of $380 \mathrm{~nm}$ to $840 \mathrm{~nm}$ that are required for solar photovoltaic generation using a plurality of pyranometers capable of measuring solar radiation in three directions such as east-west (EW), northsouth (NS), and vertical center (CE) so as to measure an incident angle of sunlight with the-highest energy density and to improve the efficiency of solar photovoltaic generation. In accordance with an aspect of the present invention, the above and other objects can be accomplished by the provision of a solar servo control tracking device using pyranometers as shown in the Figure 1.

The solar tracking system is capable of taking two signals. One signal is from the irradiation sensor and another signal is directly from the Sun. The pyranometer could measure the solar radiation from the sun and the amount of energy send to the power house. The block diagram of solar generation system for building aquarium as shown in the Figure 1. A solar panel (70); a tracker including a solar panel driving motor (71) for driving the solar panel (71); a tracker controller (100) controlling the tracker; a plurality of first quadrangular or semispherical pyranometers (10) measuring a solar azimuth in the east and west direction (EW) and detecting solar radiation of wavelengths of $380 \mathrm{~nm}$ to $840 \mathrm{~nm}$; a plurality of second quadrangular or semispherical pyranometers (20) measuring a solar azimuth in the north and south direction (NS) and detecting solar radiation of wavelengths of $380 \mathrm{~nm}$ to $840 \mathrm{~nm}$; and a third quadrangular or semispherical pyranometer (30)as shown in the Figure 1. It measuring a solar azimuth in the vertical central direction (CE) and detecting solar radiation of wavelengths of $380 \mathrm{~nm}$ to $840 \mathrm{~nm}$; where in the tracker controller (100) includes: a plurality of signal amplifiers (40) amplifying solar radiation detection signals in three azimuths of the east and west direction (EW), the north and south direction (NS), and the vertical central direction (CE), which are measured by the first, second, and third pyranometers $(10,20$, and 30); a plurality of signal processors (50) filtering the three directional amplified solar radiation detection signals by limiting a voltage; and a main control microcomputer (60) outputting a control signal to the solar panel driving motor (71) such that the solar panel (70) is stopped when the solar radiations in a first direction or the solar radiations in a second direction among the plurality of output signals in three directions output from the signal processors (50) are same and the solar radiations in one direction are maximum. The first direction where the solar radiations are same is the east and west direction (EW), the second direction where the solar radiations are same is the north and south direction (NS), and the one direction 
where the solar radiation is maximum is the vertical central direction (CE). The solar servo control tracking device using pyranometers further includes a self-diagnosis microcomputer (80) monitoring whether the output control signal of the main control microcomputer (60) is abnormal and controlling the main control microcomputer (60) to be reset or standby when the output control signal is abnormal. The signal amplifiers output voltages with respect to the three directional solar radiations detected by the first, second, and third pyranometers (10, 20, and 30) and using push-full amplifier transistors (Q1 to Q18) to the output current. The Signal processing unit as shown in the Figure 1 and each of the signal processors (50) includes: a voltage limiter (51) limiting the amplified signal of the first, second, and third pyranometers $(10,20$, and 30$)$ within $5 \mathrm{~V}$; a first input end buffer (52) buffering the voltage-limited amplified signal; a filter (53) filtering the buffered amplified signal; and a second output end buffer (54) buffering the filtered amplified signal to be output to the main control microcomputer (60). According to the present invention, energy density of wavelengths that is required for the solar photovoltaic generation can be precisely measured. Since output characteristics of the pyranometers exhibit linear log output characteristics with respect to the incident angle of sunlight, detection sensitivity is excellent to precisely detect sunlight. Due to the linear $\log$ output characteristics of the pyranometers, the incident angle of sunlight is easily detected in various weather conditions such as clear day as well as cloud weather so that efficiency of solar photovoltaic generation can be improved and sunlight can be rapidly tracked even in very changeable weather.

A solar servo control tracking device using a pyranometer according to an embodiment of the present invention, includes solar panels 70, a tracker 200 having a solar panel driving motor 71 rotating the solar panels 70; and a tracker controller 100 controlling the tracker 200.The tracker 200 is installed between a plurality of the solar panels 70 and includes the solar panel driving motor 71, a first reduction gear 72, a second worm reduction gear 73, a third worm reduction gear 74, and an azimuth sensor unit 75 .

The solar panel driving motor 71 is driven by a servo control of the tracker controller 100. To the solar panel driving motor 71 , the first reduction gear 72 , the second worm reduction gear 73 , and the third worm reduction gear 74 are sequentially connected to generate high torque required to drive the solar panels 70 that is transmitted to the solar panels 70 such that a pinion of the third worm reduction gear 74 is connected to the second worm reduction gear 73 and sequentially a worm wheel of the third worm reduction gear 73 is connected to a pinion of the third worm reduction gear 73 so as to generate maximum driving torque. A solar panel fixing shaft 76 to which the solar panels 70 are fixed is installed to the worm wheel. The azimuth sensor unit 75 is installed to the tracker 200 to detect an absolute azimuth of the solar panels 70 for the rotation angle servo control of the solar panels 70 . The azimuth sensor unit 75 includes a sensor pinion, a sensor worm wheel, and an azimuth sensor. The sensor pinion is connected to the pinion of the third worm reduction gear 74, the sensor worm wheel is connected to the sensor pinion, and the azimuth sensor is installed to a rotation shaft of the sensor worm wheel. The azimuth of the solar panels 70 detected by the azimuth sensor is transmitted to the tracker controller 100.

In the above-mentioned solar servo control tracking device, the tracker controller 100 includes pyranometers 10,20 , and 30 , a plurality of signal amplifiers 40 , a plurality of signal processors 50, the main control microcomputer 60 , and a self-diagnosis microcomputer 80 . The pyranometers 10,20 , and 30 , may be installed to sides of the solar panels 70 or at other positions.

Hereinafter, driving of the solar panels 70 by the tracker will be described using the solar panel driving motor 71 and the control of the tracker by the tracker controller 100 will be described using the plurality of signal amplifiers 40, the plurality of signal processors 50, the main control microcomputer 60, and the self-diagnosis microcomputer 80 . 
The solar servo control tracking device using a pyranometer according to the embodiment of the present invention, as illustrated in Figure 1, includes a plurality of first, second, and third pyranometers 10, 20, and 30, the plurality of signal amplifiers 40, the plurality of signal processors 50 , and the main control microcomputer 60 . The plurality of first, second, and third pyranometers 10, 20, and 30, has a quadrangular pyramid shape or a semispherical shape, and detects solar radiation of wavelengths of 380 $\mathrm{nm}$ to $840 \mathrm{~nm}$ that is required for the solar photovoltaic generation in the east and west direction EW, in the north and south direction NS, and in the vertical central direction CE.

\subsection{Design of Servo Tracking System}

The most abundant energy in the universe is obtained from sun. Hence it is the challenge for the present generation to generate maximum electricity by using solar power although many developments were made to collect the solar energy, but unable to utilize the maximum power released from sun. Hence solar tracking system has been implemented that plays a vital role in collecting the solar energy. The present invention consists of a solar servo control tracking device that performs the remote servo control and remote monitoring of solar module assemblies without manual interference according to the solar azimuth that generates maximum electric power with single power transmission that improves the tracking accuracy, with minimum cost and can check the malfunctions to track the solar luminance. The efficiency of photo voltaic power generation can be improved by $5 \%$ to $10 \%$.

The equipment is a solar servo control tracking device which consists of remotely controlling and monitoring a solar module assembly for smooth driven according to the solar azimuth. The solar azimuth is obtained from the following azimuth equation by using time $\mathrm{H}$ and declination.

\section{Simulation Results and Discussions}

In this chapter, the experimental results of the proposed solar tracking system are presented. The experimental results of the single axis solar tracking system and the dual axis solar tracking system are presented and the results of the comparison are shown.

\subsection{Design of Servo Tracking System}

Table. 1 gives the information about average development time for Fixed axis, 1 Axis and 2 Axes solar systems over a period of one year. And information considers periodically for every month. Average and ratio over one year shows that Fixed axis gives $3.67,100 \%, 1$ Axis gives $4.06,110.58 \%$ and 2 Axes gives $4.72,128.71 \%$. From these results it clearly shows that 2 Axes is better than 1 Axes and 1 Axes is better than Fixed axis.

Table 1. Single Axis, Fixed Axis and Dual Axes Tracking System Test

\begin{tabular}{|c|c|c|c|}
\hline \multirow{2}{*}{ Month } & \multicolumn{3}{|c|}{ The average development time May } \\
\cline { 2 - 4 } & $1^{\text {st }}$ system & $2^{\text {nd }}$ system & $3^{\text {rd }}$ system \\
\cline { 2 - 4 } & Fixed & 1 Axis & 2 Axes \\
\hline 1 Month & 3.553 & 3.029 & 4.503 \\
\hline 2 Month & 3.555 & 3.543 & 4.464 \\
\hline 3 Month & 4.197 & 4.354 & 5.191 \\
\hline 4 Month & 4.215 & 5.084 & 5.429 \\
\hline 5 Month & 4.350 & 5.566 & 5.892 \\
\hline
\end{tabular}




\begin{tabular}{|c|c|c|c|}
\hline 6 Month & 3.490 & 4.520 & 4.568 \\
\hline 7 Month & 3.114 & 4.116 & 4.045 \\
\hline 8 Month & 3.308 & 4.239 & 4.219 \\
\hline 9 Month & 3.870 & 4.445 & 4.881 \\
\hline 10 Month & 4.109 & 4.147 & 5.224 \\
\hline 11 Month & 3.119 & 2.901 & 4.063 \\
\hline 12 Month & 3.125 & 2.716 & 4.159 \\
\hline Average & 3.67 & 4.06 & 4.72 \\
\hline Ratio & $100.00 \%$ & $110.58 \%$ & $128.71 \%$ \\
\hline
\end{tabular}

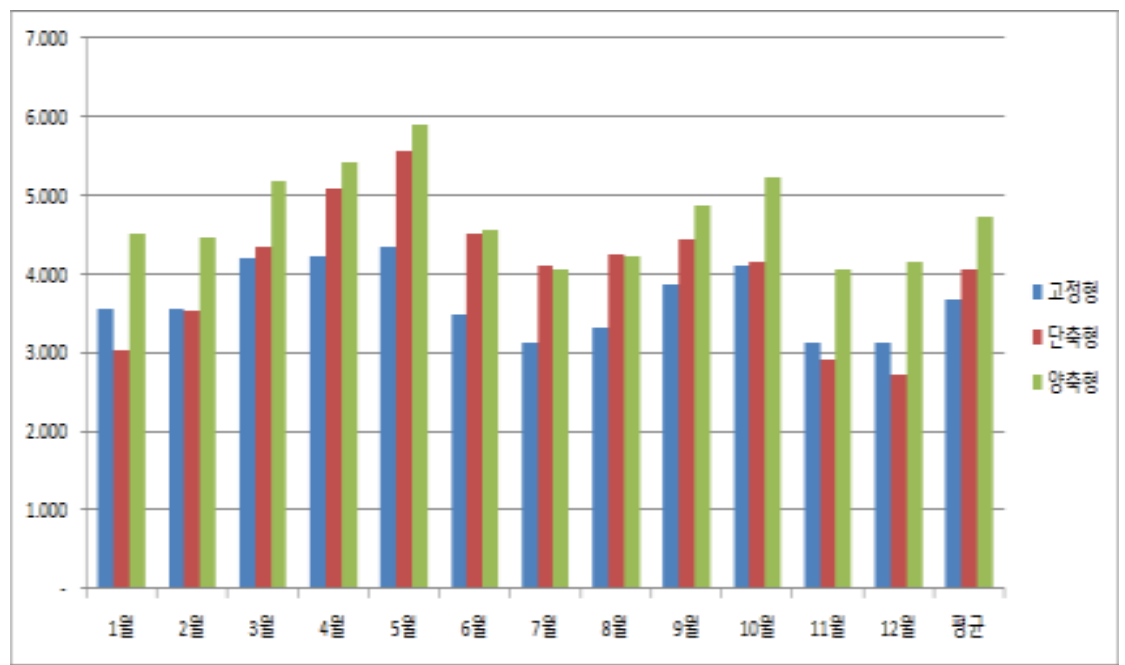

Figure 2. 1 Axis, Fixed Axis and 2 Axes Tracking System Test

\section{Conclusion and Discussion}

In this dissertation, solar tracking system and power monitoring system which are core technologies in PV power plant have been studied. centralized tracking system using a single PV cell (CTSUP) is proposed for improving efficiency of conventional system. And also String level power monitoring system (SLPMS) is proposed.

Conventional solar tracking systems are classified into two types. One is a stand-alone system using cds sensors and the other is program type. Stand-alone type using cds sensors uses cds sensors as solar sensors. Every solar tracker has a cds sensor, and each solar tracker operates independently. The program type calculates the optimum angle based on the annual solar orbit information. Based on this calculated information, the entire solar tracker operates identically. In general, a small pv power plant uses a standalone system using css sensors, and a large type pv power plant uses a program type.

Centralized tracking system using a single PV cell (CTSUP) is proposed for improving efficiency of conventional system. The proposed CTSUP basically operates similar to program type. The optimum angle of incidence can be calculated by using a small size PV cell as a solar sensor. Based on this information, it is possible to maintain the optimum angle of incidence of the PV cell at all times by correcting the tracker control information calculated in the conventional program type. CTSUP can be applied to both single axis solar tracker and dual axis solar tracker. Through various experiments, it was shown that CTSUP is more efficient than existing solar tracker system. Also, installation cost is lower than standalone tracker using cds sensor. 
String level power monitoring system which are core technologies in PV power plant have been also proposed. Important factors when designing a power monitoring system are accurate power measurement, installation cost, and fault diagnosis.

Accurate power measurement is fundamental to any type of monitoring system. In terms of price, the inverter level monitoring method has the lowest cost because it does not need a measuring device in module or string unit. The inverter level monitoring method has the lowest cost because it does not need a measuring device in module or string unit. When a particular module fails, its effects may not be known. If it is detected, it is not possible to know which string or module is faulty. In short, zone monitoring is a method with a low CAPEX and high OPEX. module-level monitoring method requires a measuring unit every single module, Installation cost, therefore, is very high. However, if an arbitrary module fails, the faulty module can be detected accurately. In short, zone monitoring is a method with a high CAPEX and low OPEX.

String level monitoring system require a measuring unit in a strings. Therefore, the string level monitoring system costs less than the module level monitoring system. In case of fault diagnosis of pv module, power monitoring method of each module can perform the most accurate fault diagnosis. On the other hand, inverter level monitoring can hardly perform fault diagnosis. In the string level monitoring system, if any module has a failure, it is easy to identify the module belonging to which string. Therefore, string monitoring is the most efficient alternative considering both price and fault diagnosis aspects.

The proposed SLPMS showed only $0.3 \%$ and $0.4 \%$ error in voltage and current measurement compared with commercial power meter. Based on this performance, even if there is a problem with only one PV panel, the corresponding string can be diagnosed immediately. The proposed fault diagnosis function of SLPMS is verified through various experiments. According to the experimental results, SLPMS recognized the difference even if only one diode in the module in the string failed due to the accurate current measurement of SLPMS. Also, the proposed fault diagnosis algorithm considers current and voltage at the same time to perform accurate diagnosis.

When the proposed CSTUP and SLPMS are simultaneously applied to the PV power plant, it is shown that not only the optimal power generation efficiency but also the initial input cost and the maintenance cost are reduced.

\section{Acknowledgments}

This work was supported by the 2016 Research Fund of University of Hanseo.

\section{References}

[1] D. H. Jang and J. S. Won, "Voltage, Frequency, and Phase-Difference Angle Control of PWM InvertersFed Two-Phase Induction Motors", IEEE Transactions on Power Electronics, vol. 9, no. 4, pp. 377-383.

[2] Y. Hori, "Future Vehicle driven by Electricity and Control-Research on Four Wheel Motored UOT Electric March II", IEEE Transaction on Industrial Electronics, vol. 51, no. 5, (2004), pp. 954-962.

[3] H. Fujimoto, A. Tsumasaka and T. Noguchi, "Vehicle Stability Control of Small Electric Vehicle on Snowy Road", JSAE Review of Automotive Engineers, vol. 27, no. 2, (2006), pp. 279-286.

[4] S. Satou and H. Fujimoto, "Proposal of Pitching Control for Electric Vehicle with InWheel Motor", IIC07- 81 IEE Japan, (2007), pp. 65-70.

[5] A. Shaoout, "Real Time System in automotive Applications: Vehicle Stability Control", Electrical Engineering Reserch, vol. 1, no. 4, (2013).

[6] Miloudi et, "Solar tracking with Photovoltaic Panel", Science Direct,Energy procedia, vol. 42, (2013), pp. 103-112.

[7] P. Papageorgas, "Smart solar panels: In-situ monitoring of photovoltaic panels based on wired and wireless sensor networks", Science Direct, Energy Procedia, vol. 36, (2013), pp. 535-545.

[8] B. Hanus, "Solar Dachanlagen", Franzis Energie technik, (2009).

[9] Y. Kumsuwan, W. Srirattanawichaikul and S. Premrudeepreechacharn, "Analysis of a two-phase induction motor using dynamic model based on Matlab /Simulink". 


\section{Authors}

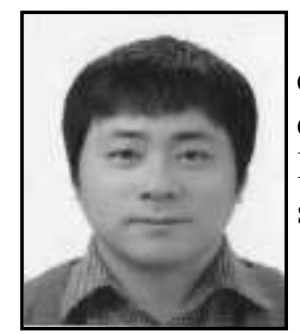

Wonchul Choi, he received his B.S. degrees in Electronic engineering at Hanseo University, Seosan, Korea in 2011, and M.S. degrees in Electronic Engineering at Hanseo University, Seosan, Korea in 2014, His current research interests include the embedded system and micro controller unit.

Hyukjae Woo, he received his B.S. and M.S. degree in Department of Electronic Engineering from Sunchon National University at 1996 and 1998. He received his Ph.D degrees in the Department of ICT Creative Convergence from Busan University of Foreign Studies at 2017, respectively. His research interests include Fault diagnosis, Solar power generation.

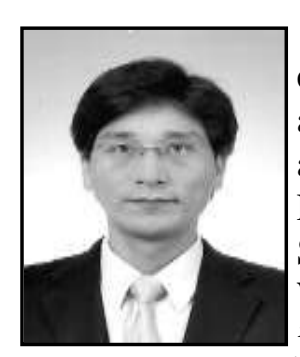

Choongchae Woo, received the B.S. degrees in Electronic engineering at Sunchon National University, Sunchon, Korea in 2000, and M.S. and Ph.D. degrees in Electrical and Electronic Engineering at Yonsei University, Seoul, Korea in 2002, and 2007, respectively. From September 2007 to February 2009, he was a senior engineer in Samsung Electronics, Suwon, Korea where he developed Mobile WiMAX systems for broadband wireless services. In March 2009, he joined the Department of Electronics Engineering at the Hanseo University as an Assistant Professor. His current research interests include the field ofwireless communications focusing on multicarrier systems and resource allocation algorithms. 